\title{
On additive bases with two elements
}

\author{
by \\ Giampiero Chiaselotti (Cosenza)
}

1. Introduction. Let $G$ be a finite abelian additive group. If $S$ is a non-empty subset, we set

$$
\Sigma(S)=\left\{\sum_{x \in B} x \mid B \subseteq S, B \neq \emptyset\right\}
$$

and

$$
X=\{m \in \mathbb{N} \mid \text { if } S \subseteq G \backslash\{0\},|S| \geq m \text { then } \Sigma(S)=G\} .
$$

Let us observe that $X \neq \emptyset$ if $|G|>2$, since $|G|-1 \in X$. The number

$$
c(G)=\min \{m \mid m \in X\}
$$

is called the critical number of $G$. It was first studied by Erdős and Heilbronn [4] for $G=\mathbb{Z}_{p}$, with $p$ a prime number. Recently the parameter $c\left(\mathbb{Z}_{q}\right)$ has been studied for various values of $q$ (see [4], [9], [1], [8]).

For evaluation of $c(G)$ for more general groups, the work of Diderrich [2] was fundamental. He proved that $p+q-2 \leq c(G) \leq p+q-1$ if $G$ is an abelian group of order $p q$, with $p, q$ prime numbers. Moreover he conjectured that $c(G)=p+h-2$ if $|G|=p h$, where $p$ is the smallest prime dividing $|G|$ and $h$ is a composite integer. First, this conjecture was checked in special cases: for $p=2$ in [3], for $p \geq 43$ in [5], and for $p=3$ in [7]. Then Gao and Hamidoune [6] gave a complete proof of the conjecture.

In additive number theory we usually ask what may be said about the set $M+M$, for a given subset $M$ of some additive structure; in particular when $M+M$ is the whole structure. In this note, if $S$ is a non-empty subset of a finite abelian group $G$, we set

$$
\Sigma_{k}(S)=\left\{\sum_{x \in B} x|B \subseteq S,| B \mid=k\right\},
$$

for any integer $k$ with $1 \leq k \leq|S|$, and we study when the subset $\Sigma_{2}(S)$ of $S+S$ is the whole $G$.

2000 Mathematics Subject Classification: 11B75, 20K09. 
For this purpose, for integer $k \leq|G|-1$ we define

$$
X_{k}=\left\{m \in \mathbb{N}|\forall S \subseteq G \backslash 0,| S \mid \geq m \Rightarrow \Sigma_{k}(S)=G\right\}
$$

and call the number

$$
c_{k}(G)=\min \left\{m \mid m \in X_{k}\right\}
$$

the $k$ th critical number of $G$. For some integer $k$ the set $X_{k}$ can be empty; in this case we set formally $c_{k}(G)=\infty$. If $k=1$ note that $c_{1}(G)=\infty$, since if $S \subseteq G \backslash\{0\}$ we have $\Sigma_{1}(S)=S \neq G$. Here we determine the 2nd critical number of any finite abelian group $G$ in terms of the order of its subgroup of elements of order 2. More precisely, if $H_{G}=\{2 g \mid g \in G\}$ and $K_{G}=\{g \in G \mid 2 g=0\}$, we prove that

$$
c_{2}(G)= \begin{cases}\left(|G|+\left|K_{G}\right|\right) / 2+1 & \text { if } H_{G} \neq\{0\} \\ \infty & \text { if } H_{G}=\{0\} .\end{cases}
$$

Finally, note that $c(G) \leq c_{k}(G)$ for any integer $k$ with $1 \leq k \leq|G|$.

2. The results. First we observe that the number $|G|+\left|K_{G}\right|$ is even. In fact, if $|G|$ is odd, then $K_{G}=\{0\}$. If $|G|$ is even, then $K_{G} \neq\{0\}$ and any element of $K_{G} \backslash\{0\}$ has order 2; therefore $\left|K_{G}\right|$ is a power of 2 .

TheOREm 2.1. Let $G$ be an abelian group of order $n$. Set

$$
H_{G}=\{2 g \mid g \in G\} \quad \text { and } \quad K_{G}=\{g \in G \mid 2 g=0\} .
$$

Then

$$
c_{2}(G)= \begin{cases}(|G|+q) / 2+1 & \text { if } H_{G} \neq\{0\} \\ \infty & \text { if } H_{G}=\{0\}\end{cases}
$$

where $q=\left|K_{G}\right|$.

Proof. Let $\phi: G \rightarrow G$ be the homomorphism $\phi: x \mapsto 2 x$; then we have $\operatorname{Ker} \phi=K_{G}$ and $\operatorname{Im} \phi=H_{G}$, therefore $G / K_{G} \cong H_{G}$. If $H_{G}=\{0\}$ then $G=K_{G}$; this implies that each element in $G \backslash\{0\}$ has order 2. In this case 0 cannot be the sum of two distinct elements of $G$; hence for each subset $S \subseteq G \backslash\{0\}$ we have $\Sigma_{2}(S) \neq G$, i.e. $c_{2}(G)=\infty$. We can therefore assume that $H_{G} \neq\{0\}$ (in this case note that $n \geq 2 q$ since $\left|H_{G}\right| \geq 2$ ).

Let $S$ be a non-empty subset of $G$ such that $0 \notin S$ and $|S| \geq(n+q) / 2+1$. Let $a \in G$. We claim that $a \in \Sigma_{2}(S)$. Let $a_{1}, \ldots, a_{m}$ be $m=(n+q) / 2+1$ distinct elements of $S$. We set

$$
A=\left\{a_{1}, \ldots, a_{m}\right\}, \quad B=a-A=\left\{a-a_{1}, \ldots, a-a_{m}\right\} .
$$

Since $|G|=n \geq|A \cup B|=|A|+|B|-|A \cap B|$, it follows that

$$
|A \cap B| \geq 2 m-n=2\left(\frac{n+q}{2}+1\right)-n=q+2 .
$$


There exist therefore $q+2$ distinct elements $a_{i_{1}}, \ldots, a_{i_{q+2}}$ of $A$ such that

$$
a_{i_{1}}=a-a_{j_{1}}, \ldots, a_{i_{q+2}}=a-a_{j_{q+2}}
$$

where $a_{j_{1}}, \ldots, a_{j_{q+2}}$ are elements of $A$. We obtain

$$
a=a_{i_{1}}+a_{j_{1}}=\ldots=a_{i_{q+2}}+a_{j_{q+2}},
$$

where $a_{i_{1}}, \ldots, a_{i_{q+2}}, a_{j_{1}}, \ldots, a_{j_{q+2}}$ are elements of $A$.

Now, if $a_{i_{k}} \neq a_{j_{k}}$ for some $k \in\{1, \ldots, q+2\}$, then $a=a_{i_{k}}+a_{j_{k}} \in \Sigma_{2}(S)$; thus now we suppose that

$$
a_{i_{1}}=a_{j_{1}}, \ldots, a_{i_{q+2}}=a_{j_{q+2}}
$$

i.e.

$$
a=2 a_{i_{1}}=2 a_{i_{2}}=\ldots=2 a_{i_{q+2}} .
$$

Set $K_{a}=\{x \in G \mid 2 x=a\}$. By (1) it follows that $K_{a} \neq \emptyset$. For every $c \in K_{a}$ the map $K_{G} \rightarrow K_{a}, x \mapsto x+c$, is onto and one-to-one; whence $q=\left|K_{G}\right|=\left|K_{a}\right|$. This implies that $G$ contains exactly $q$ distinct elements, say $y_{1}, \ldots, y_{q}$, for which $2 y_{i}=a(i=1, \ldots, q)$; but this contradicts $(1)$ since $a_{i_{1}}, \ldots, a_{i_{q+2}}$ are themselves distinct. Thus we have proved our claim. This also proves that $c_{2}(G) \leq(n+q) / 2+1$. We now want to construct a subset $S \subseteq G \backslash\{0\}$ having exactly $(n+q) / 2$ distinct elements and such that $\Sigma_{2}(S) \neq G$.

Let $a \in H_{G} \backslash\{0\}$. By definition of $H_{G}$ there exists $c \in G \backslash\{0\}$ such that $2 c=a$. If $K_{G}+c$ is the coset $\{k+c \mid k \in G\}$, we have $\left|G \backslash\left(K_{G}+c\right)\right|=n-q$ (where $n-q$ is even $\geq 2$ since $n \geq 2 q$, say $n-q=2 m$ ). We now observe that $G \backslash\left(K_{G}+c\right)$ can be partitioned into disjoint pairs of the type $\{x, a-x\}$, with $a-x \neq x$. In fact, if $x \in G \backslash\left(K_{G}+c\right)$, also $a-x \in G \backslash\left(K_{G}+c\right)$ (otherwise $a-x=k+c$ with $k \in K_{G}$ implies $x=-k-c+a=-k-c+2 c=-k+c$, which is absurd); moreover, $a-x \neq x$ (otherwise $a=2 x=2 c$ implies $2(x-c)=0$, i.e. $\left.x-c \in K_{G}\right)$. Now, since two pairs $\{x, a-x\},\{y, a-y\}$ with $x, y \in G \backslash\left(K_{G}+c\right)$ either coincide or are disjoint, we can suppose that $G \backslash\left(K_{G}+c\right)$ has the form

$$
\left\{x_{1}, \ldots, x_{m}, a-x_{1}, \ldots, a-x_{m}\right\},
$$

where $m=(n-q) / 2$.

First assume that $0 \notin\left\{x_{1}, \ldots, x_{m}\right\}$. In this case we set

$$
S=\left\{x_{1}, \ldots, x_{m}\right\} \cup\left(K_{G}+c\right) .
$$

Then $0 \notin S$ (if $0 \in K_{G}+c$, then $c \in K_{G}$ implies that $0=2 c=a$, which is impossible) and $|S|=m+q=(n-q) / 2+q=(n+q) / 2$. We prove that $a \notin \Sigma_{2}(S)$. In fact, $a \in \Sigma_{2}(S)$ if and only if one of the following conditions is satisfied: 
(i) $a=x_{i}+x_{j}$, where $i, j \in\{1, \ldots, m\}$ and $i \neq j$; but then $a-x_{j}=$ $x_{i} \in S$, and this contradicts the definition of $S$.

(ii) $a=x_{i}+(k+c)$, where $i \in\{1, \ldots, m\}$ and $k \in K_{G}$; in this case we have $x_{i}=a-k-c=2 c-k-c=-k+c \in K_{G}+c$, which is impossible.

(iii) $a=(k+c)+(\bar{k}+c)$, where $k$ and $\bar{k}$ are two distinct elements of $K_{G}$; but then $a=k+\bar{k}+2 c=k+\bar{k}+a$ implies $k+\bar{k}=0$ and since $k \in K_{G}$ we also have $k+k=0$, i.e. $k=\bar{k}$, which is absurd. Hence $a \notin \Sigma_{2}(S)$.

If $0 \in\left\{x_{1}, \ldots, x_{m}\right\}$, then $0 \notin\left\{a-x_{1}, \ldots, a-x_{m}\right\}$ and thus we set

$$
S=\left\{a-x_{1}, \ldots, a-x_{m}\right\} \cup\left(K_{G}+c\right) .
$$

In this case we also have $0 \notin S,|S|=(n+q) / 2$ and $a \notin \Sigma_{2}(S)$ (the conditions analogous to (i)-(iii) are excluded in the same way as above). Hence in both cases $a \notin \Sigma_{2}(S)$ and thus $\Sigma_{2}(S) \neq G$. This shows that $c_{2}(G) \geq(n+q) / 2+1$. Hence

$$
c_{2}(G)=\frac{n+q}{2}+1 \quad \text { if } H_{G} \neq\{0\} .
$$

COROLlary. Let $\mathbb{Z}_{n}$ be the group of integers modulo $n$, with $n>2$. Then

$$
c_{2}\left(\mathbb{Z}_{n}\right)= \begin{cases}(n+3) / 2 & \text { if } n \text { is odd }, \\ (n+4) / 2 & \text { if } n \text { is even } .\end{cases}
$$

Proof. If $n$ is odd, we have $K_{\mathbb{Z}_{n}}=\{0\}$ and $H_{\mathbb{Z}_{n}}=\mathbb{Z}_{n} \neq\{0\}$; if $n$ is even, then $K_{\mathbb{Z}_{n}}=\{0, n / 2\}$ and $H_{\mathbb{Z}_{n}} \neq\{0\}$ since $\left|H_{\mathbb{Z}_{n}}\right|=n / 2 \neq 0$. In both cases the result follows directly from Theorem 2.1.

Finally note that if $G$ is an abelian group of order $n$ and $S \subseteq G \backslash\{0\}$ has at least $\lceil n / 2\rceil+1$ elements, then $G \backslash H_{G} \subseteq \Sigma_{2}(S)$.

In fact, let $a \in G \backslash H_{G}$ and take $m=\lceil n / 2\rceil$ elements $a_{1}, \ldots, a_{m}$ in $S$. We set

$$
A=\left\{a_{1}, \ldots, a_{m}\right\} \quad \text { and } B=\left\{a-a_{1}, \ldots, a-a_{m}\right\} .
$$

Now, if $a_{i}=a-a_{j}$ for some pair $i, j$ with $i \neq j$, then $a=a_{i}+a_{j} \in \Sigma_{2}(S)$; on the other hand the condition $a_{i}=a-a_{i}$ cannot be satisfied because $a \notin H_{G}$. We can suppose therefore that $A \cap B=\emptyset$. Then

$$
|A \cup B|=|A|+|B|=2\left\lceil\frac{n}{2}\right\rceil \geq n
$$

implies that $A \cup B=G$. Hence the remaining element of $S \backslash A$ must be contained in $B$. This proves that $a \in \Sigma_{2}(S)$, i.e. $G \backslash H_{G} \subseteq \Sigma_{2}(S)$.

\section{References}

[1] J. A. Dias da Silva and Y. O. Hamidoune, Cyclic subspaces of Grassmann derivations, Bull. London Math. Soc. 26 (1994), 140-146. 
[2] G. T. Diderrich, An addition theorem for abelian groups of order $p q$, J. Number Theory 7 (1975), 33-48.

[3] G. T. Diderrich and H. B. Mann, Combinatorial problems in finite abelian groups, in: A Survey of Combinatorial Theory, J. L. Srivasta et al. (eds.), North-Holland, Amsterdam, 1973, 95-100.

[4] P. Erdős and H. Heilbronn, On the addition of residue classes $\bmod p$, Acta Arith. 9 (1964), 149-159.

[5] W. Gao, On the size of additive bases of finite groups, preprint, 1997.

[6] W. Gao and Y. O. Hamidoune, On additive bases, Acta Arith. 88 (1999), 233-237.

[7] Y. O. Hamidoune, A. S. Lladó and O. Serra, On sets with a small subset sum, Combin. Probab. Comput. 8 (1999), 461-466.

[8] E. Lipkin, Subset sums of sets of residues, Astérisque 258 (1999), xiii, 187-193.

[9] J. E. Olson, An addition theorem modulo p, J. Combin. Theory 5 (1968), 45-52.

Dipartimento di Matematica

Università della Calabria

87036 Arcavacata di Rende (Cosenza)

Italy

E-mail: chiaselo@unical.it

Received on 3.8.2000

and in revised form on 18.12.2000 